\title{
Genome-guided discovery of natural products through multiplexed low coverage whole-genome sequencing of soil Actinomycetes on Oxford Nanopore Flongle
}

Rahim Rajwani ${ }^{1}$, Shannon I. Ohlemacher ${ }^{1}$, Gengxiang Zhao ${ }^{1}$, Hong-bing Liu ${ }^{1}$, Carole A. Bewley ${ }^{1 \#}$

${ }^{1}$ Laboratory of Bioorganic Chemistry, National Institute of Diabetes and Digestive and Kidney Diseases, National Institutes of Health, Bethesda, Maryland 20892, United States

Correspondence to Dr. Carole A. Bewley (caroleb@nih.gov) 


\section{Abstract}

Genome-mining is an important tool for discovery of new natural products; however, the number of publicly available genomes for natural product-rich microbes such as Actinomycetes, relative to human pathogens with smaller genomes, is small. To obtain contiguous DNA assemblies and identify large (ca. 10 to greater than $100 \mathrm{~Kb}$ ) biosynthetic gene clusters (BGCs) with high-GC (>70\%) and -repeat content, it is necessary to use long-read sequencing methods when sequencing Actinomycete genomes. One of the hurdles to long-read sequencing is the higher cost.

In the current study, we assessed Flongle, a recently launched platform by Oxford Nanopore Technologies, as a low-cost DNA sequencing option to obtain contiguous DNA assemblies and analyze BGCs. To make the workflow more cost-effective, we multiplexed up to four samples in a single Flongle sequencing experiment while expecting low-sequencing coverage per sample. We hypothesized that contiguous DNA assemblies might enable analysis of BGCs even at low sequencing depth. To assess the value of these assemblies, we collected high-resolution mass-spectrometry data and conducted a multiomics analysis to connect BGCs to secondary metabolites.

In total, we assembled genomes for 20 distinct strains across seven sequencing experiments. In each experiment, $50 \%$ of the bases were in reads longer than $10 \mathrm{~Kb}$, which facilitated the assembly of reads into contigs with an average N50 value of $3.5 \mathrm{Mb}$. The programs antiSMASH and PRISM predicted 629 and 295 BGCs, respectively. We connected BGCs to metabolites for $N, N$-dimethyl cyclic-ditryptophan, a novel lassopeptide and three known Actinomycete-associated siderophores, namely mirubactin, heterobactin and salinichelin.

\section{Importance}

Short-read sequencing of GC-rich genomes such as Actinomycetes results in a fragmented genome assembly and truncated biosynthetic gene clusters (often 10 to $>100 \mathrm{~Kb}$ long), which hinders our ability to understand the biosynthetic potential of a given strain and predict the molecules that can be produced. The current study demonstrates that contiguous DNA assemblies, suitable for analysis of BGCs, can be obtained through low-coverage, multiplexed sequencing on Flongle, which provides a new low-cost workflow (\$30-40 per strain) for sequencing Actinomycete strain libraries. 
Clinical pathogens are increasingly becoming resistant to currently used antimicrobials causing over 700,000 deaths worldwide (1). New antimicrobials are urgently needed to alleviate antimicrobial resistance and prevent deaths per year to rise over 10 million by 2050 (1). One of the prolific sources of new antimicrobials is a group of gram-positive mycelia forming bacteria, the Actinomycetes. Several currently used antibiotics, including vancomycin, rifamycin, and streptomycin are isolated from Actinomycetes and they still hold enormous potential for the future discovery of new medicines (2).

Genome sequencing is now an important component of natural products research. Whole-genome sequencing (WGS) enables identification of the genes responsible for the biosynthesis of natural products (3). Often genes required for the biosynthesis of a natural product positionally cluster on the genome and are referred to as biosynthetic gene clusters (BGCs) (4). The BGC sequences can be used to predict possible structures of the resulting natural product (5), assess novelty of the compound (6) and dereplicate compounds from a strain collection (7). Despite the merits offered by WGS, the number of Actinomycete genomes remains limited. Several rare genera are not represented by a complete genome, and the majority of currently available genomes are sequenced using Illumina short-read technology that results in highly fragmented assemblies. BGCs span multiple contigs in fragmented genome assemblies and cannot be detected or analyzed by commonly used BGC prediction tools such as antiSMASH $(8,9)$.

Long-read sequencing technologies (e.g. PacBio or Oxford Nanopore Technologies, ONT) produce contiguous genome sequences needed to analyze secondary metabolite gene clusters. Notably, PacBio assemblies achieve consensus accuracy over $99.999 \%$; however, it is generally less accessible due to the upfront cost of sequencing instruments and higher per sample sequencing costs. By contrast, ONT does not require an upfront cost of an expensive sequencing instrument and the devices are inexpensive. Nevertheless, ONT data results in a lower consensus accuracy (99.9\%) and often requires polishing with Illumina reads to obtain reference-quality genomes. We hypothesized that while BGC identification requires a contiguous DNA sequence, it might be less affected by the lower consensus accuracy of a Nanopore assembly since most BGC analysis steps involve inferring homology between distantly related amino acid sequences using profile Hidden Markov models. If this is true, contiguous DNA assemblies can be obtained at ca. 10x coverage using ONT, allowing complete genome sequencing at a significantly lower cost. While such ONT sequenced genomes would still require error correction with Illumina reads, they could be used on their own to sequence a strain collection, build a catalog and compare BGCs for dereplication or identification of potentially new compounds, which might be particularly useful to natural product research and drug discovery programs.

To assess the feasibility of obtaining contiguous assemblies from ca. 10x sequencing depth, predicting BGCs, and connecting BGCs to metabolites, we conducted the current multi-omics study. We sequenced 20 new soil-derived Actinomycete strains and analyzed their metabolome using high-resolution mass spectrometry (HRMS). For sequencing, we specifically selected Flongle, a recently launched ONT

\section{8} sequencing device that costs $\$ 90$ USD and can generate up to 1-2 Gigabases of sequence output. With a typical Actinomycetes genome being 8-10 Mb, a single Flongle experiment might be sufficient to sequence 3-4 strains at 20-30x coverage. Sequencing workflows based on Flongle could be broadly applicable to small and large studies due to the modular experimental design. In the current study, we 
obtained 300-850 $\mathrm{Mb}$ of data per experiment across ten sequencing experiments with read-length N50 values over $10 \mathrm{~Kb}$. Assembling of reads resulted in contiguous assemblies (average contig N50 value = $3.5 \mathrm{Mb}$ and average number of contigs $=47.3$ ). AntiSMASH5 predicted a total of 629 BGCs from these assemblies. Through a combined analysis with metabolomics data, we were able to connect BGCs to their secondary metabolites. The study demonstrates the utility of low coverage nanopore-only assemblies as a rapid and low-cost sequencing option to advance natural product research.

Results

\section{An in silico analysis to study the effect of sequencing coverage and read length on BGC} detection

We first analyzed what level of sequencing coverage would be sufficient for contiguous assemblies and BGC detection using Oxford Nanopore sequencing. For this purpose, three Actinomycete genomes, previously sequenced at high coverage, were downloaded from the European Nucleotide Archive and their reads were down sampled to 60x, 30x, 15x and 7x coverage (assuming a genome size of $8 \mathrm{Mb}$ ) before assembling and detecting BGCs. While actual genome sizes of the three genomes differed (Table $\mathrm{S} 1$ ), an assumption of a fixed expected genome size of $8 \mathrm{Mb}$ allowed us to determine the utility of a prospective sequencing experiment where Actinomycete genome sizes would not be known. In the down sampling analysis, assembly size and number of predicted genes nearly plateau at ca. $15 \mathrm{x}$ coverage. Similarly, a sharp decline in the number of contigs and number of mismatches per $100 \mathrm{~Kb}$ was observed at ca. 15-20x coverage (Figure 1). At approximately the same coverage of 15-20x, 72-96\% of BGCs were detected by antiSMASH and a further increase in coverage led to detection of only 1-6 additional BGCs (Figure 1). Moreover, most of the BGCs were not located at the edge of a contig, also referred to as complete. Relative to antiSMASH, PRISM predicted a lower number of BGCs. This could be because antiSMASH was run in a 'relaxed' mode in this study whereas PRISM does not have this option. Nevertheless, the trend relative to coverage was similar between antiSMASH and PRISM.

Assembly contiguity and therefore BGC detection in a Nanopore sequencing experiment is also related to read length. In another computational experiment, we evaluated whether longer reads might enable a more contiguous DNA assembly and BGC detection at fixed coverage. For this purpose, simulated Nanopore reads of average length $500,1000,2000$ and 4000 were generated at 10x coverage of a Streptomyces genome (GB4-14) using BadRead. The resulting reads were assembled and analyzed for assembly contiguity and BGC detection. It was observed that an ca. 2-fold increase in average read length was associated with a 2-fold reduction in the number of contigs (Figure $\mathrm{S} 3$ ). Improved assembly contiguity also led to a reduction in the number of BGCs on contig edges (incomplete) and an increased number of complete BGCs with little to no change of sequencing coverage (Figure S 4 ).

Overall, these computational experiments suggested contiguous DNA assemblies and complete BGCs can be detected at low sequencing coverage using long reads from Oxford Nanopore Technologies; this should allow for a dramatic reduction in cost per genome through multiplexing. The computational experiments were followed up with prospective sequencing of Actinomycetes genomes using Flongle and more detailed analyses of BGCs described in the following sections. 


\section{Nanopore sequencing, genome assembly and quality assessment}

A total of ten sequencing experiments were conducted- each with an attempt to sequence four Actinomycetes strains (Figure 2). Impurities in the starting genomic DNA (as measured by a ratio of the UV absorbance at 260 and $280 \mathrm{~nm}$ ) and low pore occupancy (caused by insufficient loading of the library or inhibition of adapter ligation) resulted in three unsuccessful experiments with a total output $<100$ megabases (Mb) per experiment. The remaining seven experiments yielded 288- $797 \mathrm{Mb}$ over 18-24 hours. The longest read for each sample was over $80 \mathrm{~Kb}$.

Across the experiments, we tried different buffers for bead-based purification to apply size selection and increase the read length N50 values from standard protocols (Table S2) (Figure 2). One of our initial experiments using $0.5 \mathrm{x}$ of the standard buffer concentration was not successful resulting in read N50 values of 1-1.6 Kb for three out of five samples sequenced in the experiment. In two subsequent successful experiments (AET670and AFK704), we utilized 0.15x of a modified buffer containing $0.5 \mathrm{M}$ $\mathrm{MgCl}_{2}+5 \%$ PEG in TE buffer (10 mM Tris-Cl pH 8.0, $1 \mathrm{mM}$ EDTA) for bead-based purification after barcodes ligation as described previously (10). Read length N50 values in these experiments were 11.6$15.1 \mathrm{~Kb}$ (Figure 2). In three experiments (AEZ324, AFA498 and AFA876), the concentration of the modified buffer-based size selection was reduced to $0.1 \mathrm{x}$ which led to a further increase in read length N50s (10.5-23.3 Kb) accompanied by an increased sample loss. Application of size selection after barcodes ligation ensures approximately equal fragment lengths for pooling of samples and adapter ligation. However, ligation of barcodes could be less efficient to longer fragments if shorter fragments are present in the mixture. We tested buffer-based size selection ( $0.1 \mathrm{x}$ beads in modified buffer) before barcode ligation in later experiments (flow cell ids AFK426 and AFK406) (Table S 2). A more consistent output was observed, possibly due to more efficient barcode/adapter ligation to longer DNA fragments.

Across the seven successful runs, 3,814,434,062 bases in 751,459 reads were generated. Upon demultiplexing, the median number of bases per sample was $77.5 \mathrm{Mb}$ (theoretical coverage of $9.5 \mathrm{x}$ with an expected $8 \mathrm{Mb}$ genome size). Three strains were sequenced at $<2.5 x$ theoretical coverage $(<20 \mathrm{Mb}$ per strain) and were excluded from further analysis. Subsequently, 25 samples (20 distinct isolates) were de novo assembled with Canu and polished with Racon and medaka (Figure 3). The median length of the obtained assemblies was $8.5 \mathrm{Mb}$ (average: 7.9 Mb, maximum: $9.4 \mathrm{Mb}$ ), typical of Actinomycete genome size. The only exception was a $3 \mathrm{Mb}$ assembly for GB8-002 which was also sequenced at the least coverage (4.0x) (Figure 3).

We assessed the accuracy and quality of these low coverage genomes by comparing them with genomes sequenced at high coverage on MinION or PacBio. In particular, two strains, GA3-008 and GB4-14 were previously sequenced by our lab at 10-fold higher coverage using MinION and PacBio, respectively (Table 1). Despite the lower sequence coverage, the genomes' contiguity was only slightly affected on flongle and all were assembled into $<10$ contigs. The size of the assembly differed by $6.1 \mathrm{~Kb}$ (GA3-008) and $19.6 \mathrm{~Kb}$ (GB4-14) due to insertion/deletion (indel) errors. Despite many mismatches and indel errors, $87.5-100 \%$ of the BGCs detected in the MinION or PacBio assemblies were also detected in these Flongle assemblies by antiSMASH.

\section{Taxonomy and BGCs}

antiSMASH predicted 629 BGCs of 29 different types across all assembled genomes from this study (Figure S 5). Seventy-nine percent (497/629) of these BGCs were complete (i.e. not located on a contig 
edge). There was a median of 23 BGCs per strain. The number of non-ribosomal peptide synthases (NRPSs) and terpenes detected were 2-3 times greater overall than other BGC types; however, this may be because antiSMASH does not subdivide these BGC types into defined sub-classifications, like it does for the RiPPs (LAP, lanthipeptide, etc.) and PKS (type I, II, etc.) categories. In addition to antiSMASH, 295 BGCs were predicted using PRISM software. A unique feature of PRISM is that it enables chemical structure prediction from BGCs (11). In the current dataset, PRISM generated a predicted chemical structure for 180 out of 295 predicted BGCs.

The taxonomic identification based on 16S rRNA sequences extracted from the WGS revealed that the dataset comprises 11 different Actinomycete species belonging to four genera (Table S 3 ). It consisted of eight Amycolatopsis, nine Streptomyces, four Lentzea and four Nocardia species. Some species were overrepresented in the dataset. Amycolatopsis lurida and Streptomyces tendae were each represented with four strains and Lentzea violacea with two strains (Table S 3). The biosynthetic diversity between strains was high with members of the same species, sharing $>99 \%$ identity in their 16 S sequences, differing by up to 20 BGCs (Figure S 6). The biosynthetic diversity of BGCs within species also varied in some cases. For instance, strains of Streptomyces tendae, Lentzea violacea or Streptomyces kanamyceticus were more diverse within the species than strains of Amycolatopsis lurida. Different species of the same genus also encoded 10-15 different BGCs on average.

\section{Predicting metabolites from BGCs - paired analysis of genome and secondary metabolites}

\section{Insilco PRISM-predicted chemical structures}

The PRISM predicted BGCs detected from low coverage assemblies were analyzed further to determine whether they could be linked to a metabolite. We conducted a paired analysis by collecting MS/MS spectra for extracts from strain cultures grown in ISP1 and R2A media. MS/MS spectra were queried using molDiscovery against a database of PRISM predicted chemical structures from BGCs (12). The database comprised 1,177 structures generated from 180 BGCs sequenced in this study. A total of 18 predicted structures matched to MS/MS spectra collected from the strains at false discovery rate (FDR) $<1 \%$ and $p$-value $<\mathrm{e}^{-10}$. Three of these matches were also detected in media blanks and were excluded from the analysis. These three metabolites corresponded to tryptophan, and the dipeptides Pro-Val and Phe-Val.

Two of eighteen matched structures were predicted from a cyclic dipeptide BGC in Amycolatopsis strain GA6-002 and were detected in metabolite extracts from the same strain (Figure 4). The two gene BGC encoded a cyclic dipeptide synthase (CDPS) and an $N$-methyl transferase. Both of the amino acyl tRNA binding pockets of the CDPS had a specificity signature for tryptophan tRNA. Based on sequence information, cyclic-di-tryptophan c(WW) was predicted as a possible metabolite that can be methylated on nitrogen by the $\mathrm{N}$-methyl transferase as reported previously for Actinosynnema mirum (13). In all chemical extracts from GA6-002, a metabolite matching the precursor $m / z$ and predicted MS/MS fragmentation pattern for $\mathrm{c}(\mathrm{WW})$ was detected. In addition, in the ethyl acetate extracts from ISP1 cultures the $\mathrm{N}$-methylated metabolite $\mathrm{c}(\mathrm{WW}) \mathrm{Me} 2$ was also detected. 
An additional mass spectral match was detected for a small, glycosylated polyketide in GB4-14 consisting of a propionate unit and an actinosamine sugar moiety (Figure $S$ 7), resembling a putative shunt product of a larger polyketide. The corresponding BGC includes additional PKS modules that were not accounted for in the structure predicted by PRISM. More complex structures that better resemble final products of PKS pathways were predicted from a more contiguous Flongle (Figure 3 ) or PacBio assembly (Table 1 ) of the same strain but were not detected in the metabolite extracts. The final product of this BGC, predicted from PacBio sequenced genome, was therefore regarded as not detected.

\section{RiPPs}

We conducted a second analysis to query MS/MS spectra for post-translationally modified precursor peptides from RiPPS using MetaMiner (14). All open reading frames shorter than $600 \mathrm{nt}$ were extracted from 43 antiSmash predicted RiPP BGCs (16 lanthipeptide, 4 LAP, 13 lassopeptides, 10 thiopeptides) and included in this analysis (Figure $\mathrm{S} 5$ ). We observed a single high confident match for a class-II lassopeptide BGC in the Amycolatopsis sp. GA6-002 (Figure 5). The BGC encoded all essential elements for lasso peptide biosynthesis including precursor peptide, asparagine synthetase (SMCOG1177 essential for macrolactam formation), lassopeptide transglutamase protease (PF13471 - leader peptide cleavage) RiPP recognition element (PF05402), and ABC transporter (SMCOG1288 and SMCOG1000) (15, 16)(Figure 5) $[14,15]$. The precursor $m / z\left(1041.504[\mathrm{M}+2 \mathrm{H}]^{2+}\right.$ and $\left.694.672[\mathrm{M}+2 \mathrm{H}]^{3+}\right)$ of the matched spectra was consistent with the predicted core peptide after loss of one water molecule (-18.010). The $\mathrm{MS}^{2}$ fragmentation pattern further indicated abundant ions matching $\mathrm{m} / \mathrm{z}$ for $\mathrm{y} 6$ and $\mathrm{y} 7$ ions. The 16 amino acid core peptide sequence (GYPWWDNRDIFGGRTFL) is a novel lassopeptide variant with $76 \%$ amino acid identity to propeptin, an endopeptidase inhibitor (17). The analysis was also repeated for RiPP BGCs predicted by PRISM and no matches were detected with $p$-value lower than $\mathrm{e}^{-10}$.

\section{Known metabolites and their BGCs}

An important application of genome sequencing is to understand the biosynthesis of known natural products. Similarity to characterized BGCs can also be used for strain dereplication. To assess this application on the current sequencing data, we screened MS/MS spectra for known natural products in the Natural Product Atlas database (3) (29,006 compounds) using molDiscovery (12). Subsequently, we analyzed genome sequences to confirm the presence of the corresponding BGCs. A total 324 significant matches to known compounds were detected $\left(p\right.$-value $\left.<e^{10}\right)$. Of these, 30 had a reference MS/MS spectrum available in GNPS. We compared the spectra observed in our dataset to the reference spectra available in GNPS and found highly similar MS/MS spectra (Figure S 8).

Twenty-one of the 324 identified compounds had a previously characterized BGC in the MiBiG database (4). Twelve of these were known Actinomycete natural products; therefore, a higher sequence similarity could be expected. The other nine were compounds isolated from diverse bacterial genera, including those from Gram-negative bacteria and the phylum Cyanobacteria. The presence in our genomes of homologous BGCs for four known Actinomycetes compounds could be confirmed using BLAST sequence similarity searches (Figure 6). These compounds included $\mathrm{N}$-acetyl tryptophan and the siderophores heterobactin A, mirubactin and salinichelin. From the BGC comparisons illustrated in Figure 6, it is evident that the nanopore-sequenced genomes from this study can have sequencing artifacts resulting in fragmentation of large genes into multiple small ORFs (see for example the comparison of 
mirubactin). However, matches to homologous BGCs in MiBiG were easily identified by the high sequence identity between genes, shared functions, and synteny.

\section{BGCS encoding known antibiotic classes with no metabolites detected - glycopeptide, aminoglycoside and aminocoumarin}

In addition to the above-described BGCs whose metabolites were expressed and detected by HRMS/MS, many other BGCs with sequence homology to known antibiotic BGCs were also identified in the sequencing data. However, we were unable to assign metabolite products to these BGCs by LCHRMS/MS data. A few such BGCs are described below.

Three Amycolatopsis lurida strains (GB15-009, GA10-003 and GA10-004) harbored a nearly identical aminocyclitol gene cluster which encoded a homolog of 2-epi-5-epi-valiolone synthase (salQ) responsible for the first step in the biosynthesis of C7N-aminocyclitols (18) (Figure S 9). Aminocyclitols are biosynthesized from sugars through cyclization by a Sugar Phosphate Cyclase (SPC) such as dehydroquinate (DHQ) synthase. The BGCs were highly homologous to cetoniacytone A sharing $70-82 \%$ amino acid identity for core biosynthetic genes $(19,20)$.

In Amycolatopsis coloradensis B06-03, an aminocoumarin BGC was detected (Figure S 10). Aminocoumarin antibiotics are biosynthesized from L-tyrosine (21). Tyrosine is activated by an adenylation domain and covalently attached to a peptidyl carrier protein (PCP). A NovH-like cytochorome P450 hydroxylates PCP-bound tyrosine to $\beta$-hydroxy tyrosyl-S-PCP. A 3-oxoacylacylcarrierprotein (ACP) reductase converts it to a $\beta$-keto-tyrosyl intermediate that undergoes cyclization to form 3-amino-4,7-dihydroxycoumarin. In B06-03, downstream core aminocoumarin biosynthesis genes, a type-I polyketide BGC encoding co-enzyme A ligase (CAL) domain specific for 3amino-5-hydroxybenzoic acid (AHBA), was present as in rubradirin (22) and chaxamycin (23).

A total of seven BGCs similar to previously characterized glycopeptide BGCs were detected (Figure S 11). Glycopeptides are biosynthesized through a multi-modular NRPS assembly line. Glycopeptide BGCs encode additional tailoring enzymes such as P450 monoxygenases and glycosyltransferases that result in amino acid crosslinking and glycosylation respectively, to yield the complex multicyclic antibiotics exemplified by vancomycin. The expected glycopeptides from four glycopeptide-like BGCs (from strains GA10-004, GA10-003, GB8-002, GB15-009) were similar in amino acid composition to ristocetin (24). These four strains primarily contained butylated hydroxytoluene (Bht), dihydroxyphenylglycine (Dhpg) and 4-hydroxyphenylglycine ( $\mathrm{Hpg}$ ) as seen in ristocetin. The predicted glycopeptide from B06-03 is predicted to contain Trp, Hpg and Tyr as seen in complestatin (25). 
Soil Actinomycetes hold enormous potential for the discovery of new antibiotics. However, the number of genome-sequenced Actinomycetes in the public domain is still limited, partly due to the cost of longread next-generation sequencing. In this study, we assessed the capability of the ONT Flongle platform as a low-cost sequencing option to obtain multiple near-complete genomes of Actinomycetes, identify BGCs, and connect them to metabolites through a paired genome-metabolome analysis.

Our sequencing and assembly results showed that up to four near-complete genomes of Actinomycete strains could be sequenced on a single Flongle device. Skipping an optional DNA fragmentation step enabled read lengths up to $80 \mathrm{~Kb}$ in each sample. Bead-based size selection further depleted shorter DNA fragments, and enriched sequencing reads in longer sequences $(10 \mathrm{~Kb}+)$. The long reads enabled contiguous DNA assemblies at lower sequencing depth. The size of the assembly was typical of soil Actinomycetes, suggesting that assemblies represent near-complete genomes of the strains. There were several mismatches in the accuracy comparison analysis in flongle genomes relative to PacBio or highcoverage MinION genomes. However, the contiguity of the genomes was only slightly affected (1-2 contigs verses 6-7 contigs), indicating that important structural information about the genome (e.g., position and organization of genes) can be inferred from these sequences.

A common strategy to obtain contiguous and accurate genome assemblies is through polishing contiguous nanopore assemblies with Illumina reads. One of the significant findings of this study is that BGC predictions and their connection to metabolites was performed without the need for errorcorrection using Illumina reads. Based on down sampling analysis of public datasets, it was initially hypothesized that low-coverage nanopore-only assemblies could be used to predict and analyze BGCs. Through prospectively sequencing Actinomycetes using Flongle, it was empirically evaluated in the current study.

An interesting observation on BGC analysis was that active site specificity for various BGC classes (NRPS, PKS and CDPS) in the Flongle assemblies were correctly predicted. The active site specificities were used by PRISM to generate possible structures, which were then used to query MS/MS data for potential spectral matches. A spectrum match to a predicted structure indirectly proves that active site specificities were correct, for instance, in the case of cWW. However, we also observed that frameshifts and sequencing errors affected in silico prediction of accurate structures for some BGCs.

The analysis of RiPP BGCs in flongle assemblies was relatively less affected by sequencing. RiPP metabolite prediction is based on short precursor peptide and BGC prediction relies on detecting posttranslational modifying enzymes through error-tolerant profile Hidden Markov models. The chance of a mismatch underlying a 100 nucleotide (30-mer core peptide sequence) is low. For example, 19,227 mismatches were detected in total in a flongle assembly relative to PacBio, which corresponds to a chance of less than one mismatch per $100 \mathrm{nt}(19,227$ mismatches $/ 7183038 \mathrm{nt}$ genome size $\times 100 \mathrm{nt}=$ 0.26 per $100 \mathrm{nt}$ ). This is evident through accurate prediction of a new lassopepetide BGC and its corresponding experimental mass spectrum in the extract from strain GA6-002.

301 Similarly, BGCs homologous to previously characterized BGCs for known metabolites can be identified 302 through sequence similarity searches. The consensus accuracy of the assemblies was observed to be $30399.5 \%$ accurate, which makes a genome sequence suitable for comparison with known BGC sequences 
or to compute average nucleotide identify with published genomes. We demonstrated this through the rediscovery of BGCs and selected Actinomycetes siderophores.

While our results suggest Flongle is a useful platform for sequencing Actinomycetes, increased consistency in total sequencing output might enable further optimized workflows. For instance, Flongle flow cells were less consistent in the number of starting pores ( $<60$ out of an expected 126 in most cases), which affected total sequencing output and lower than desired coverage for a few samples. A consistent and anticipated number of pores $(>100)$ across experiments would allow for higher sequencing coverage using the same experimental workflow or allow more genomes to be sequenced in an experiment.

While a few BGCs could be connected to the metabolites in the current study, most remained unconnected. Connecting BGCs to metabolites is a multi-factor problem not limited by sequencing accuracy alone. Improvements in experiments and computational algorithms would be needed to circumvent this issue in the future. First, it is highly unlikely that all BGCs will be expressed when strains are grown in only two culture media as tested here; thus, additional media and growth conditions or genetics-free elicitor screens should be used (26-28). Second, only PRISM in silico predicted structures were used. In the future, a more extensive in silico structure generation that addresses ambiguous active site specificities (e.g., two or more possible amino acids at a site in NRPS) could be used. Third, MS/MS data were queried for exact compound spectral matches. Minor differences between predicted and expressed metabolites (e.g., single-site methylation or hydroxylation) would result in a mass shift, and a match would not be possible.

In summary, multiplexed low coverage sequencing of Actinomycetes genomes on Flongle is a promising option for the genome-guided discovery of natural products. Numerous research laboratories house valuable bacterial strain collections (29-34). Limited by the costs of large-scale long read sequencing, genome sequencing of natural product producing bacteria usually occurs on a strain-by-strain basis \{Sun, 2021 \#295;Li, 2021 \#316;Yang, 2021 \#317;Braesel, 2018 \#326\}. The future of natural product research is expected to involve analysis of genomics and metabolomics data using genome mining (e.g. antiSMASH and PRISM) and mass spectrum matching tools (such as molDiscovery integrated with NPAtlas-like databases used in this study). Indeed, such efforts are already taking place on metagenomic data sets $(38,39)$; while those studies provide vast amounts of data on the natural products-ome, a key limitation is that the data are not connected to archived bacterial strains. It is our hope that low-cost sequencing workflows such as the one described here may allow for access to genome sequencing on a larger scale and/or to a broader community of researchers, especially in resource-limited settings. 


\section{Materials and Methods}

\section{Strain isolation}

340

341

342

343

The twenty sequenced strains were a subset of streptomycin, novobiocin or vancomycin resistant strains from an in-house Actinomycetes strain library housed in the Laboratory of Bioorganic Chemistry, National Institutes of Health. The strains were isolated from soil specimens collected from deserts in Arizona, California and Nevada through standard procedures described in a previous study (35).

\section{Nanopore sequencing}

Each strain was cultivated for 3-7 days in $10 \mathrm{~mL}$ of Tryptic Soy broth (BD Diagnostic, catalog no. 211768) with $0.5 \%(\mathrm{w} / \mathrm{v})$ glycine from frozen glycerol stocks. The cultures were centrifuged at $10,000 \mathrm{xg}$ for 10 minutes and cell pellets were resuspended into $250 \mu \mathrm{L}$ Tris EDTA (TE) buffer followed by addition of 50 $\mu \mathrm{L}$ of lysosome $(100 \mathrm{mg} / \mathrm{ml})$. The mixture was incubated overnight $(16 \mathrm{hrs})$ at $37^{\circ} \mathrm{C}$. The next morning $10 \mu \mathrm{L}$ of RNase $\mathrm{A}(10 \mathrm{mg} / \mu \mathrm{L})$ was added to the cell lysate and incubated for an additional 20 min after which $250 \mu \mathrm{L}$ of proteinase $\mathrm{K}(400 \mu \mathrm{g} / \mu \mathrm{L})$ was added and incubated for 2 hours. DNA was purified from cell lysates using 1:1 v/v phenol-chloroform extraction and the DNA was collected from the upper phase. Genomic DNA was precipitated with 0.7 volume of isopropanol, washed with $80 \%$ ethanol, and resuspended into $50 \mu \mathrm{LTE}$.

DNA libraries were prepared using Oxford Nanopore Ligation Sequencing Kit (SQK-LSK109) and the native barcoding kit (NBD104) protocol for Flongle with some modifications. A DNA fragmentation step was not performed. $500 \mathrm{ng}$ of genomic DNA was directly processed for DNA end repair with NEBNext Ultra II End repair/dA-tailing Module (New England Biolabs, catalog no. E7546). Barcodes were ligated to the end-repaired DNA and purified with $0.1 \mathrm{x}$ or $0.15 \mathrm{x}$ beads (Omega Bio-Tek Inc, catalog no. M1378-01), resuspended in a custom buffer (10 mM Tris-Cl pH 8.0, $1 \mathrm{mM}$ EDTA, $0.5 \mathrm{M} \mathrm{MgCl}_{2}$ and 5\% PEG). A pooled library was prepared by combining $62.5 \mathrm{ng}$ of each barcoded DNA. Nanopore adapters were ligated to the pooled library followed by library loading and sequencing according to the manufacturer's instructions.

\section{Data-dependent untargeted LC-MS/MS}

Each strain was cultivated in deep well plates containing $400 \mu \mathrm{L}$ of ISP1 (BD Diagnostic, catalog no 276910) or R2A media (Teknova, catalog no. R0005). The cultures were incubated at $30^{\circ} \mathrm{C}$ with shaking at $200 \mathrm{rpm}$ for one week before extraction with an equal volume of ethyl acetate followed by extraction with $n$-butanol. Uninoculated media were used as blanks / negative control, and any metabolite observed in a blank run was excluded from interpretation. The LC-MS/MS data was collected using an Agilent 1290 Infinity II UPLC system equipped with an Agilent 6545 qTOF mass spectrometer. Samples were chromatographed on a Agilent Eclipse Plus C18 2.1x50mm column ( $3 \mu \mathrm{L}$ injections) using a gradient of $99 \%$ A ( $0.1 \%$ formic acid in water) to $95 \%$ B (acetonitrile) at a flow rate of $0.5 \mathrm{~mL} / \mathrm{min}$ over $10 \mathrm{~min} . \mathrm{MS} / \mathrm{MS}$ fragmentation was carried out in auto mode with collision energies of 10, 20 and $40 \mathrm{KeV}$ excluding precursor ions in the range of $40-180 \mathrm{~m} / \mathrm{z}$ and abundance below 7,000 counts.

\section{Data analysis}




\section{Genomics}

Primary genomic data analysis was conducted by basecalling with guppy (version 4.2.2, model dna_r9.4.1_450bps_hac.cfg), demultiplexing with Qcat (version 1.0.6) and assembling with Canu (version 2.0) (40). Canu assemblies were constructed with an expected genome size of $8 \mathrm{Mb}$, minimum read length threshold of $1 \mathrm{~Kb}$, minimum coverage of 2, and high Mhap error correction sensitivity (40). The genome assemblies were polished by aligning reads to the assembly and calling consensus with Racon and Medeka (41). Genes and secondary metabolite gene clusters were predicted using the programs antiSMASH (version 5) and PRISM (version 4.4.5) (11).

\section{Assessing effect of sequencing coverage on BGC detection}

FastQ reads for previously sequenced Actinomycete genomes were downloaded from the European Nucleotide Archive, dowsampled to an estimated coverage of 60x, 30x, 15x and 7x using seqtk (assuming an $8 \mathrm{Mb}$ genome as in prospective sequencing) (Table $\mathrm{S} 1$ ). Seqtk allows random subsampling of reads. Reads were subsampled to desired coverage according to the following estimation: number of reads for $Q$ coverage $=(Q /$ original coverage $) \times$ original number of reads. The downsampled Fast $Q$ files were assembled with Canu, polished with Medeka and BGCs predicted using antiSMASH. The number of mismatches in each assembly relative to original coverage was calculated using Quast (42). For consistency with data from this study (presented in Figure 3), the coverage presented is aligned coverage, taking into account the size of the final assembly and not the expected size (i.e. $8 \mathrm{Mb}$ ). The mapped coverage was extracted from Canu assembler tig information files.

\section{BGC comparison between strains}

The antiSMASH-predicted BGC sequences were extracted from each strain's genome assemblies and aligned in all possible strain pair combinations using minimap2, allowing for $5 \%$ sequence divergence (43). If a BGC from strain-1 did not align to any of the BGCs in strain-2, it was considered absent in strain-2.

\section{Homologous BGCs of previously characterized metabolites}

Homologous BGCs of previously characterized metabolites were obtained through the "known cluster blast' module of antiSMASH. The output of the program contains gene-wise blast hits for each BGC in the genome to BGCs in MiBiG (4). To identify the best hit in MiBiG database, output was first filtered to obtain MiBiG BGCs that share the largest number of genes, highest mean percent identity and highest mean coverage with genes in the query BGC. The results were subsequently filtered to retain only BGCs where the ratio of lengths between query and MiBiG BGCs was between 0.7 to 1.1.

\section{LC MS/MS Analysis}

Analysis of LC MS/MS data was conducted by conversion of the vendor.d format to mzXML files using the GNPS conversion utility. These mzXML files were subsequently used for all analyses.

\section{Spectrum matching - known or unknown structures}

\section{Spectrum matches for known metabolites using the Natural Product Atlas or unknown metabolites} (PRISM predicted structures) were identified by using molDiscovery $(3,12)$. molDiscovery computes theoretical MS/MS spectra of compounds in the database, identifies spectrum matches at user-defined mass-tolerance, and subsequently calculates statistical significance by matching the spectrum against a decoy database. In this analysis, mass tolerance of 20 ppm, p-value less than $\mathrm{e}^{-10}$ and FDR less than $1 \%$ were considered. 
417 RiPPs

418 Spectrum matches for RiPPs were detected using metaminer (14). Given a list of short peptides,

419 metaminer constructs possible RiPP products based on knowledge of post-translational modifications

420 within RiPPs. It then predicts an MS/MS spectrum for each predicted RiPP product and conducts a

421 search of experimentally collected MS/MS spectra for potential matches. All open-reading-frames

422 (ORFs) shorter than $600 \mathrm{nt}$ (200 amino acids) located within RiPP BGCs predicted by antiSMASH or

423 PRISM were used for this analysis.

424 Integration of the mass spectrometry and genomic sequences was achieved through R scripts and

425 several packages including MSnbase (44) and Open Babel (45).

426 Data availability

427 Raw sequencing data are available under NCBI Project accession no: PRJNA752621. Genome assemblies

428 and additional data are available at Figshare (https://figshare.com/articles/dataset/ /15094044 ). The

$429 \mathrm{MS} / \mathrm{MS}$ spectra have been uploaded to GNPS with accession number: MSV000087950. Scripts used in

430 data analysis and preparation of figures are available at

431 https://github.com/rajwanir/flongle actinomycetes paper.

432

433

\section{Acknowledgements}

434 This work was supported by the NIH Intramural Research Program (NIDDK) and utilized the

435 computational resources of the NIH HPC Biowulf cluster (http://hpc.nih.gov). 
bioRxiv preprint doi: https://doi.org/10.1101/2021.08.11.456034; this version posted August 12, 2021. The copyright holder for this preprint (which was not certified by peer review) is the author/funder. All rights reserved. No reuse allowed without permission. 
bioRxiv preprint doi: https://doi.org/10.1101/2021.08.11.456034; this version posted August 12, 2021. The copyright holder for this preprint (which was not certified by peer review) is the author/funder. All rights reserved. No reuse allowed without permission.

\section{Figures}



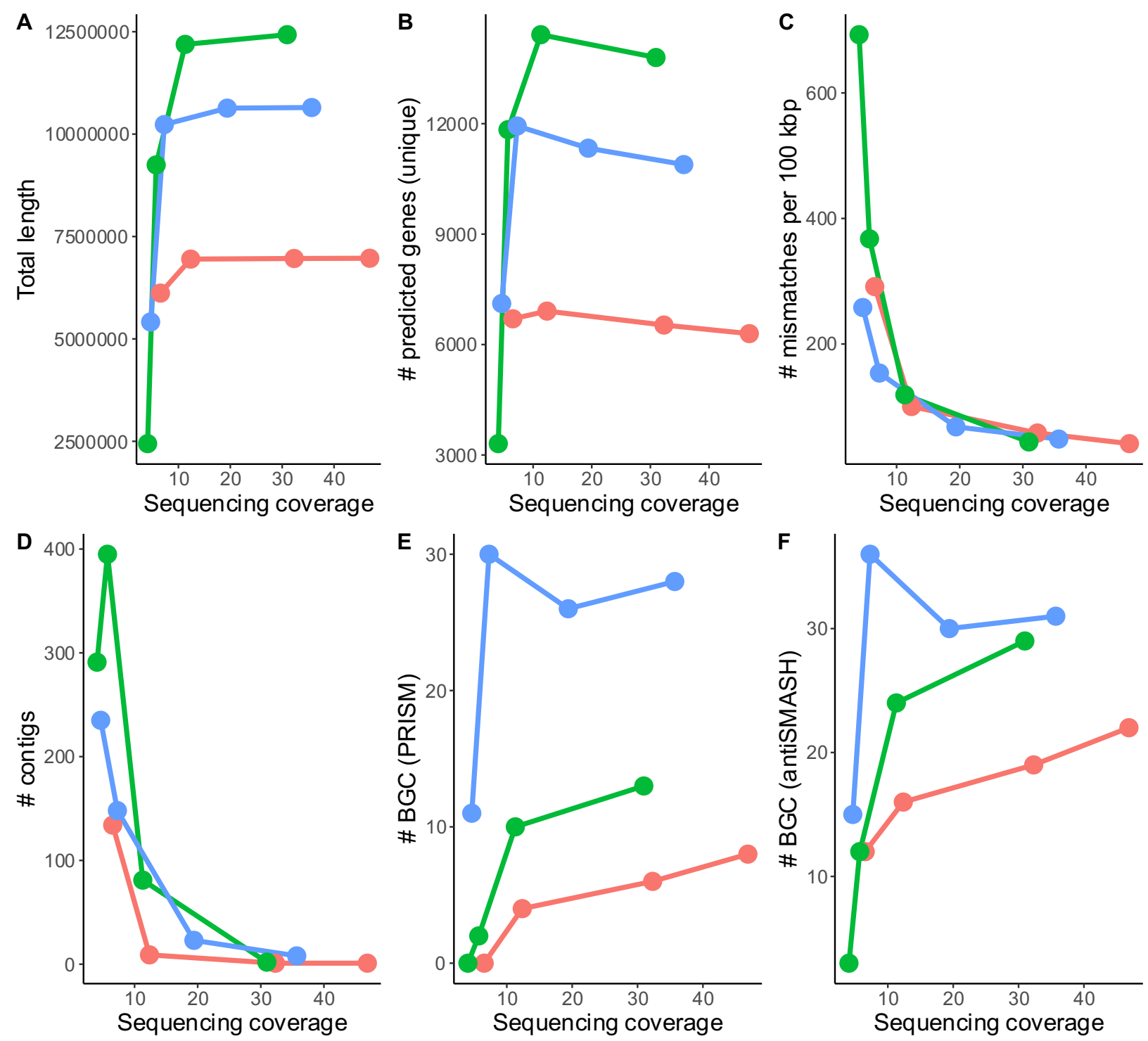

Figure 1: An in silico analysis of the effect of sequencing coverage on assembly quality and BGC prediction.

Three genomes previously sequenced at high coverage on ONT MinION/GridION platform were down sampled to the indicated sequencing coverages and then assembled. A-F The quality of the assembly as indicated by total assembly length, number of contigs, number of mismatches per $100 \mathrm{~Kb}$ and number of unique predicted genes are shown along with the number of BGCs predicted by antiSmash. The sequencing data for this analysis was downloaded from the European Nucleotide Archive. Accession numbers: 1 =SRR10597857, 2 = SRR9710049, $3=$ DRR240480). 


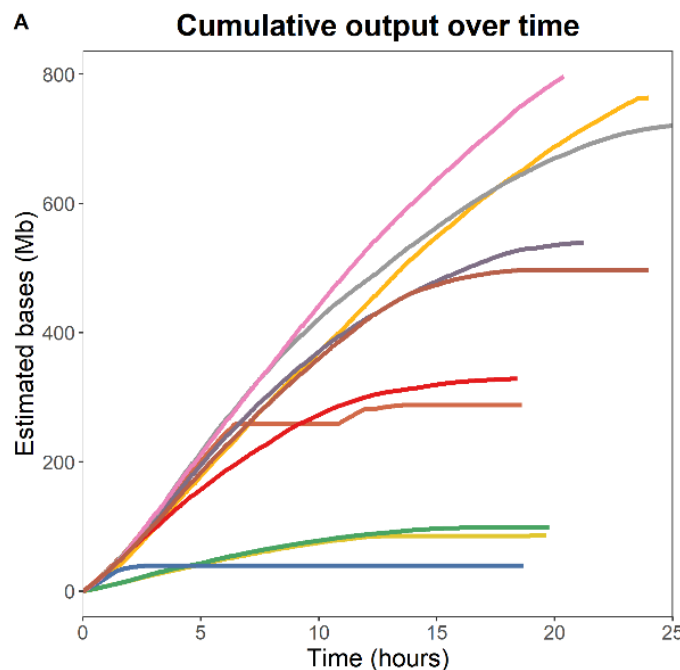

C Read length distribution

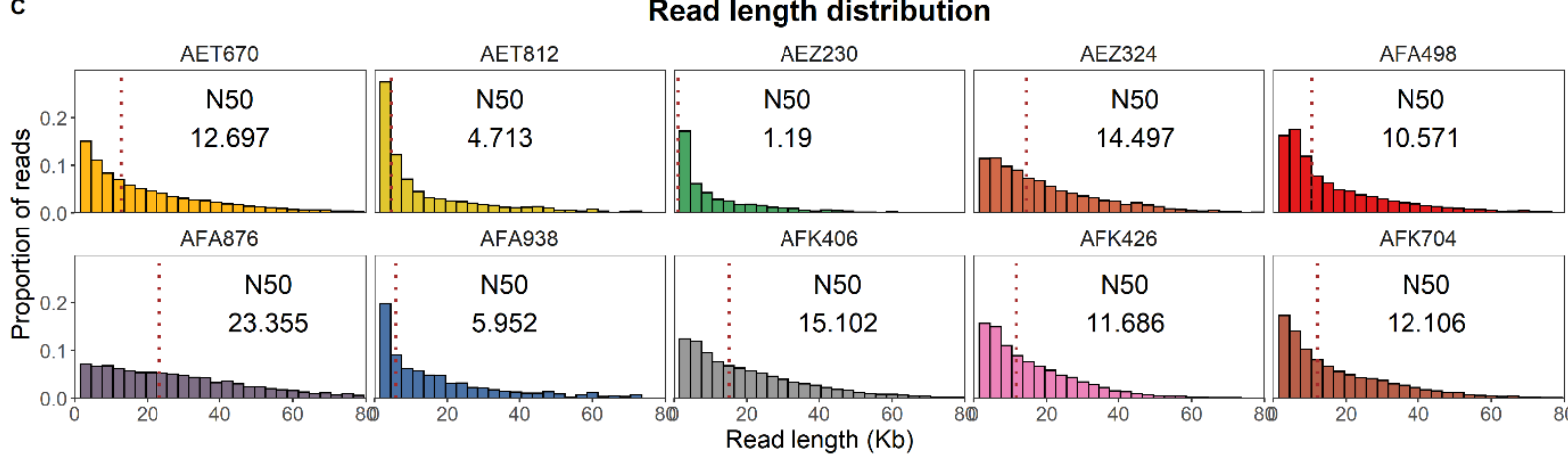

Pore activity over time

Purity of starting DNA
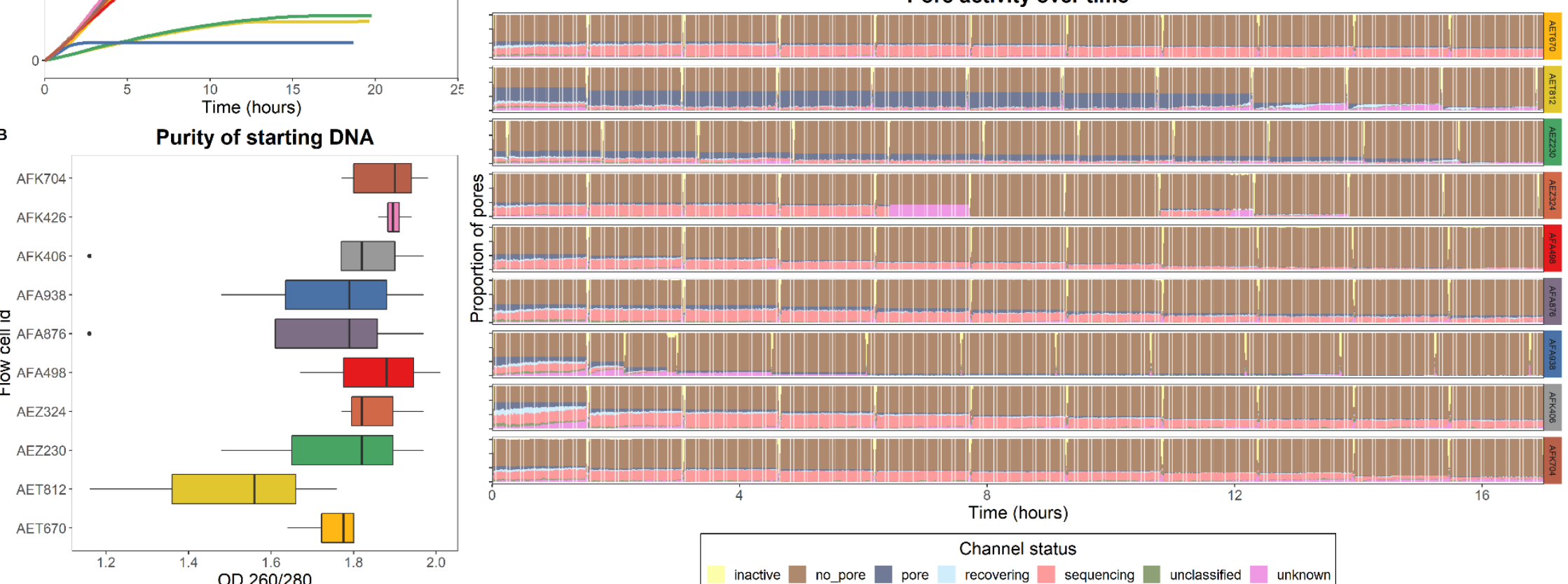

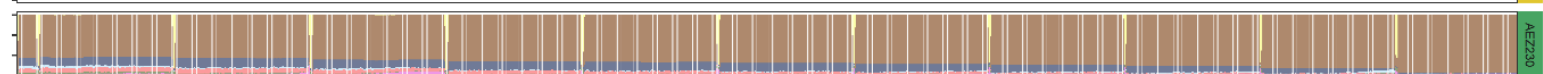

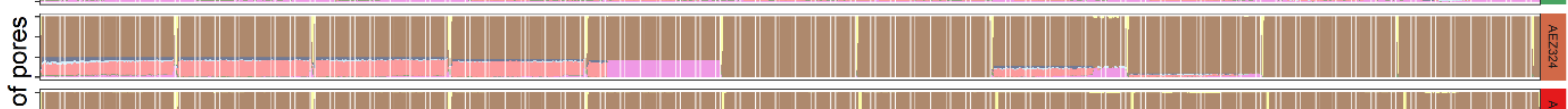

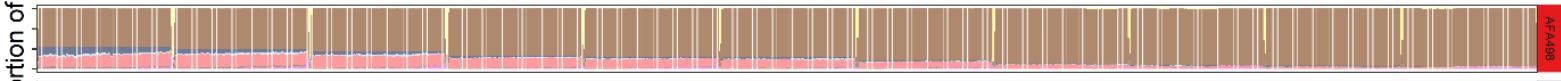

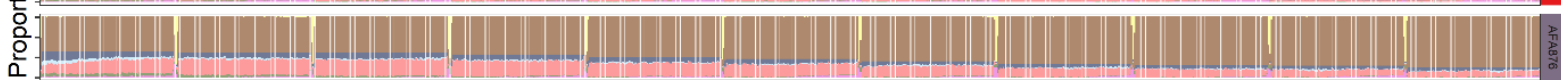

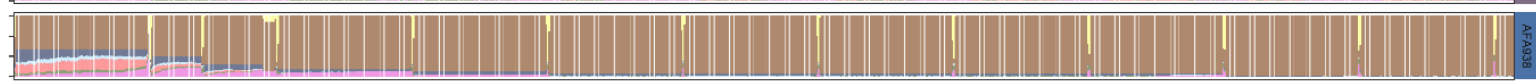

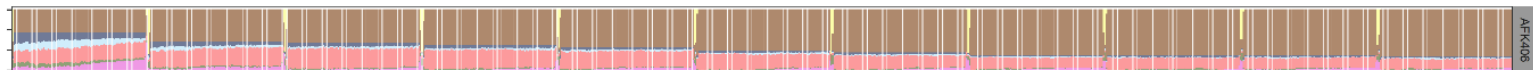

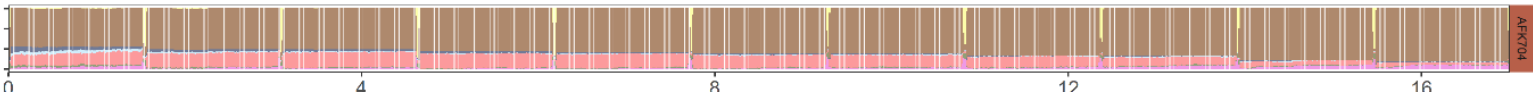
Time (hours)

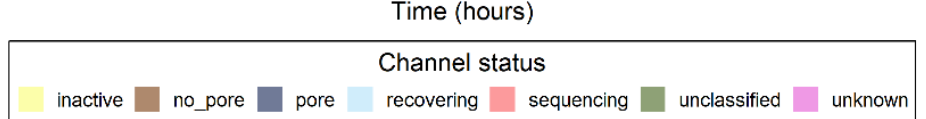

Figure 2: Library preparation and sequencing quality metrics.

The data in each panel (A-D) are colored and grouped by flow cell as indicated in panel B. Panel A indicates cumulative output (estimated megabases) over time (hours) across 10 sequencing experiments. Panel B indicates the purity (nanodrop 260/280 ratio) for samples run in each experiment. Panel C indicates read length distribution across experiments. Read N50 value (50\% of bases are 
in reads longer this value) in each experiment is labeled. Panel $D$ shows run performance at the pore level in each experiment except AFK4226. The AFK4226 experiment was interrupted at the end and the instrument did not generate the pore activity metadata to include in this chart. "Sequencing" indicates that the pore is occupied with DNA and is sequencing. "Pore" indicates an empty pore with no DNA, "no pore" indicates an inactive pore (i.e. unavailable for sequencing). 


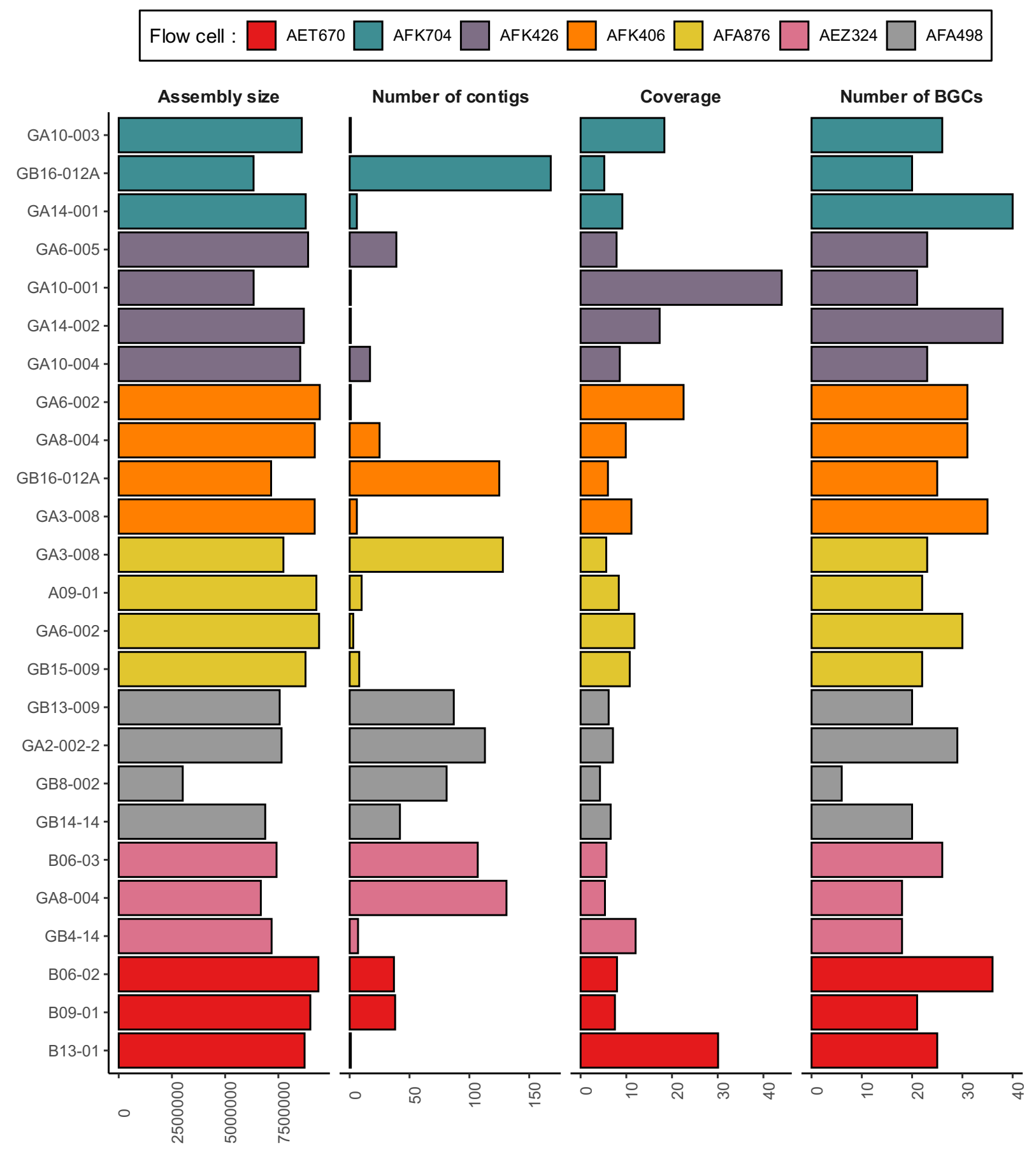

Figure 3: Characteristics of the genome assemblies obtained through low-pass multiplexed sequencing on Flongle. Each bar represents a sample. Bars are grouped and colored by flow cells (individual sequencing experiment). 
A

Biosynthetic gene cluster

ctg1_2887

ctg1_2888

Gene function

B

Amide $\mathrm{N}$-methyltransferase $\square$ Cyclodipeptide synthase

Predicted structure

cWW-Me2

cWW<smiles>CN1C(=O)C(Cc2c[nH]c3ccccc23)C(=O)N(C)C1Cc1c[nH]c2ccccc12</smiles><smiles>O=C1NC(Cc2c[nH]c3ccccc23)C(=O)NC1Cc1c[nH]c2ccccc12</smiles>

C Extracted ion chromatogram

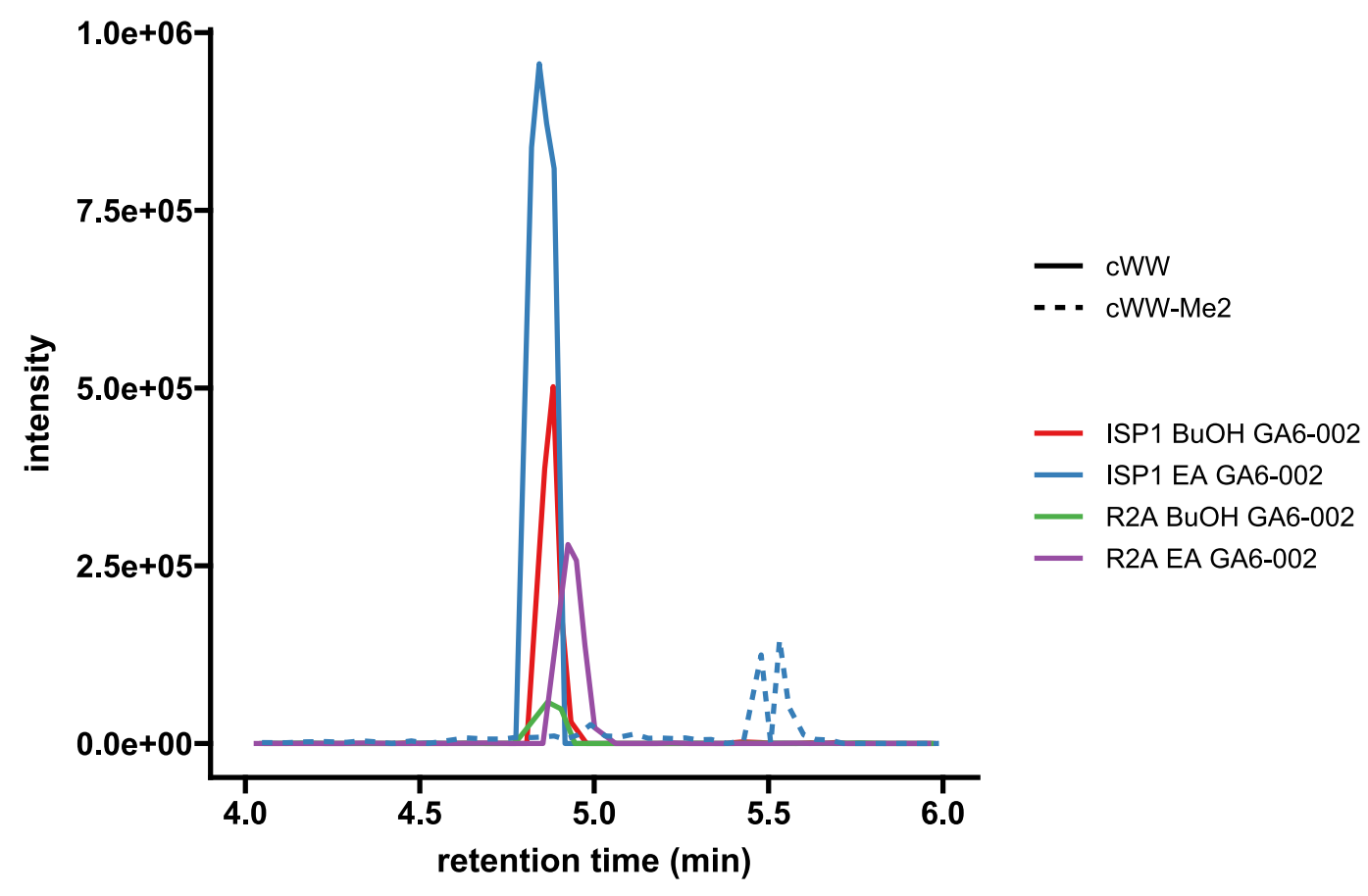

Figure 4: An N-methylated cyclo(Trp-Trp) cyclic dipeptide detected in Amycolatopsis sp. GA6002 and its corresponding BGC. A) Cyclic dipeptide BGC and B) the structures predicted by PRISM based on predicted specificity of the cyclic dipeptide synthase. C) Extracted ion chromatogram for cyclic di tryptophan (cWW) and its $N$-methylated derivative (cWW-Me2) observed in four separate crude extracts. 


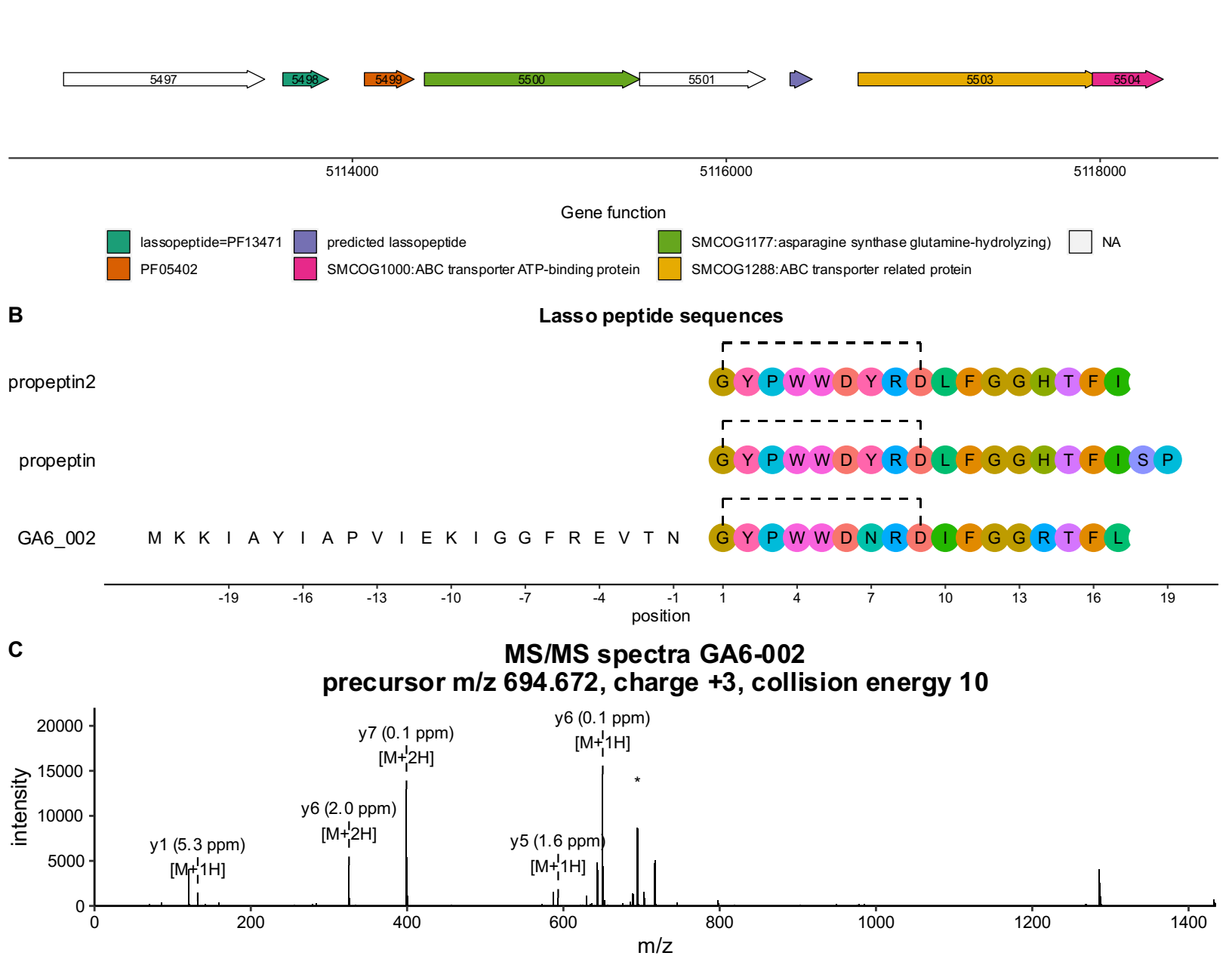

Figure 5: A new lasso peptide variant identified in GA6-002.

A) Lasso peptide biosynthetic gene cluster. Genes are colored according to their functions. B) Lassopeptide sequences in propeptin2, propeptin and GA6-002 (this study). Amino acid positions for leader sequence are shown as negative numbers. A BGC for propeptin 2 and propeptin has yet to be characterized and therefore the leader sequences are not shown for them. Dashed line between Gly and Asp shows the position of crosslink. C) MS/MS spectra that matches to the post-translationally modified predicted core sequence in GA6-002. 

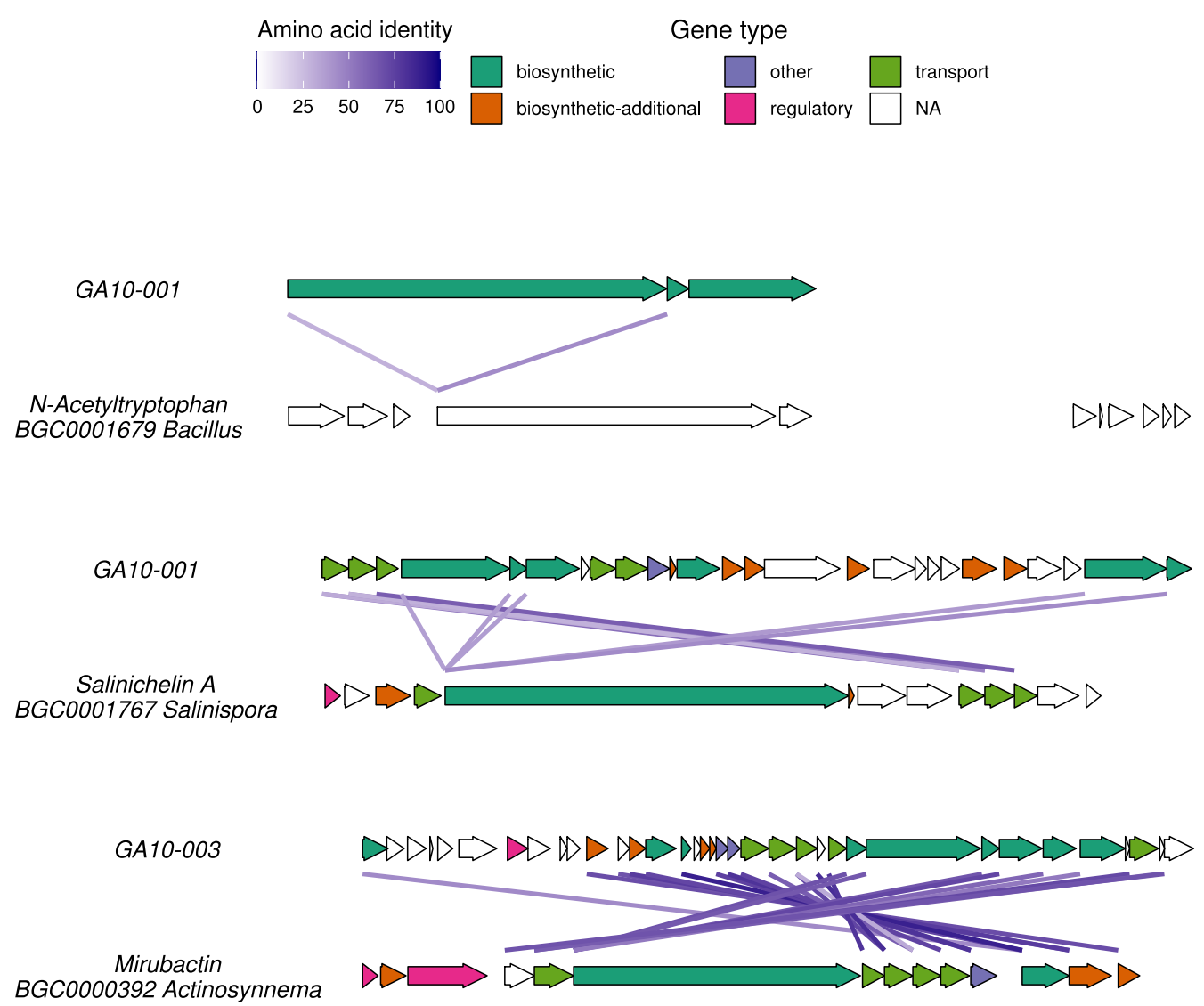

GA14-001

Heterobactin A BGC0000371 Rhodococcus
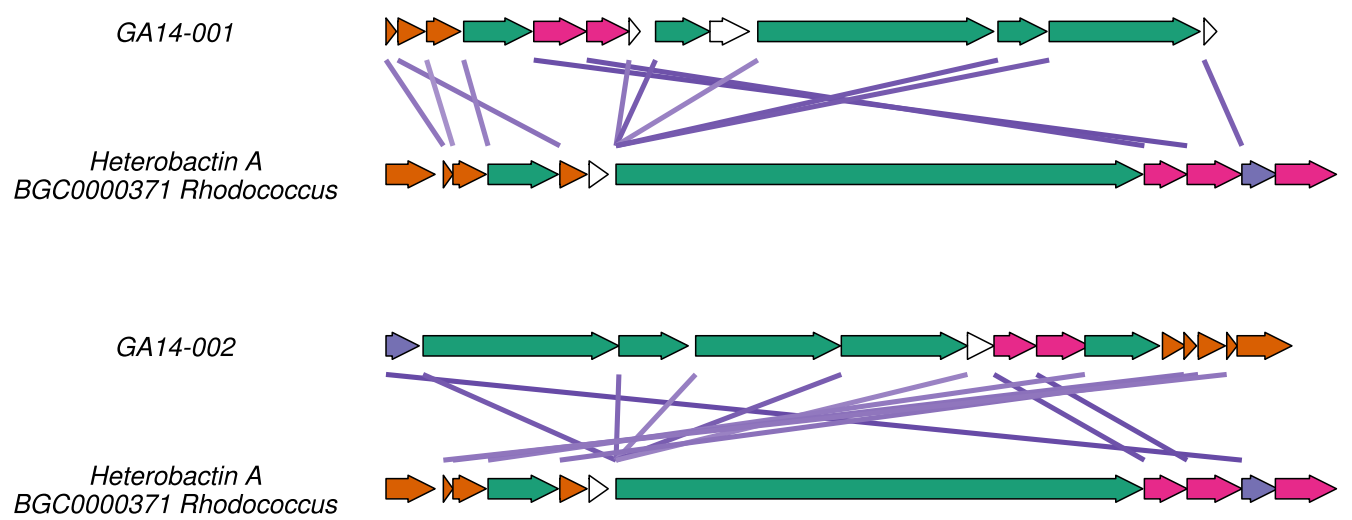

Figure 6: Homologous BGCs mapped for known metabolites detected in LC-MS/MS.

Alignments of BGCs in the MiBiG database to those identified in a producing strain from this study. Genes are colored according to function. Lines between genes indicate similarity between genes with its color intensity proportional to the BLAST amino acid identity. 
Tables

Table 1: Quality assessment of genomes of two strains (GA3-008 and GB4-14) obtained from low coverage flongle assemblies, compared to PacBio and MinION assemblies.

\begin{tabular}{lllll}
\hline Attribute & \multicolumn{3}{c}{ GA3-008 } & \multicolumn{2}{c}{ GB4-14 } \\
& $\begin{array}{l}\text { Flongle } \\
\text { multiplex }\end{array}$ & $\begin{array}{l}\text { Minion } \\
\text { singleplex }\end{array}$ & $\begin{array}{l}\text { Flongle } \\
\text { multiplex }\end{array}$ & PacBio \\
Est. coverage & 15.3 & 121.65 & 14 & 200 \\
\# contigs & 6 & 1 & 7 & 2 \\
$\begin{array}{l}\text { Assembly } \\
\text { size }\end{array}$ & 9205503 & 9199325 & 7183038 & 7163416 \\
\# & 3000 & - & & \\
mismatches* & & & 19227 & - \\
\# indels * & 13803 & - & 14484 & - \\
\# BGCs & 35 & 40 & 18 & 18 \\
\hline
\end{tabular}

* Number of mismatches and indels in the Flongle multiplexed assemblies are relative to the same strain sequenced by either MinION or PacBio. 


\section{References}

1. Organization WH. 2019. No time to wait: Securing the future from drug-resistant infections. World Health Organization: Geneva, Switzerland.

2. Genilloud O. 2017. Actinomycetes: still a source of novel antibiotics. Nat Prod Rep 34:1203-1232.

3. van Santen JA, Jacob G, Singh AL, Aniebok V, Balunas MJ, Bunsko D, Neto FC, Castaño-Espriu L, Chang C, Clark TN, Cleary Little JL, Delgadillo DA, Dorrestein PC, Duncan KR, Egan JM, Galey MM, Haeckl FPJ, Hua A, Hughes AH, Iskakova D, Khadilkar A, Lee J-H, Lee S, LeGrow N, Liu DY, Macho JM, McCaughey CS, Medema MH, Neupane RP, O’Donnell TJ, Paula JS, Sanchez LM, Shaikh AF, Soldatou $S$, Terlouw BR, Tran TA, Valentine M, van der Hooft JJJ, Vo DA, Wang M, Wilson D, Zink KE, Linington RG. 2019. The Natural Products Atlas: an open access knowledge base for microbial natural products discovery. ACS Cent Sci 5:1824-1833.

4. Kautsar SA, Blin K, Shaw S, Navarro-Muñoz JC, Terlouw BR, van der Hooft JJJ, van Santen JA, Tracanna V, Suarez Duran HG, Pascal Andreu V, Selem-Mojica N, Alanjary M, Robinson SL, Lund G, Epstein SC, Sisto AC, Charkoudian LK, Collemare J, Linington RG, Weber T, Medema MH. 2019. Mibig 2.0: A repository for biosynthetic gene clusters of known function. Nucl Acids Res 48:D454D458.

5. Navarro-Muñoz JC, Selem-Mojica N, Mullowney MW, Kautsar SA, Tryon JH, Parkinson El, De Los Santos ELC, Yeong M, Cruz-Morales P, Abubucker S, Roeters A, Lokhorst W, Fernandez-Guerra A, Cappelini LTD, Goering AW, Thomson RJ, Metcalf WW, Kelleher NL, Barona-Gomez F, Medema MH. 2020. A computational framework to explore large-scale biosynthetic diversity. Nat Chem Biol 16:60-68.

6. Kloosterman AM, Cimermancic P, Elsayed SS, Du C, Hadjithomas M, Donia MS, Fischbach MA, van Wezel GP, Medema MH. 2020. Expansion of RiPP biosynthetic space through integration of pangenomics and machine learning uncovers a novel class of lantibiotics. PLoS Biol 18:e3001026.

7. Ganley JG, Pandey A, Sylvester K, Lu K-Y, Toro-Moreno M, Rütschlin S, Bradford JM, Champion CJ, Böttcher T, XU J, Derbyshire ER. 2020. A systematic analysis of mosquito-microbiome biosynthetic gene clusters reveals antimalarial siderophores that reduce mosquito reproduction capacity. Cell Chem Biol 27:817-826.

8. Medema MH, Blin K, Cimermancic P, de Jager V, Zakrzewski P, Fischbach MA, Weber T, Takano E, Breitling R. 2011. Antismash: Rapid identification, annotation and analysis of secondary metabolite biosynthesis gene clusters in bacterial and fungal genome sequences. Nucl Acids Res 39:W339W346.

9. Meleshko D, Mohimani H, Tracanna V, Hajirasouliha I, Medema MH, Korobeynikov A, Pevzner PA. 2019. Biosyntheticspades: Reconstructing biosynthetic gene clusters from assembly graphs. Genome Res 29:1352-1362.

10. Stortchevoi A, Kamelamela N, Levine SS. 2020. SPRI beads-based size selection in the range of 2-10 kb. J Biomol Tech doi:10.7171/jbt.20-3101-002:jbt.20-3101-3002.

11. Skinnider MA, Johnston CW, Gunabalasingam M, Merwin NJ, Kieliszek AM, MacLellan RJ, Li H, Ranieri MRM, Webster ALH, Cao MPT, Pfeifle A, Spencer N, To QH, Wallace DP, Dejong CA, Magarvey NA. 2020. Comprehensive prediction of secondary metabolite structure and biological activity from microbial genome sequences. Nature Commun 11:6058.

12. Hosein M, Liu C, Mustafa G, Azat T, Alexey G. 2021. MolDiscovery: Learning mass spectrometry fragmentation of small molecules. Nature Comm doi:10.21203/rs.3.rs-71854/v1.

13. Giessen TW, von Tesmar AM, Marahiel MA. 2013. A trna-dependent two-enzyme pathway for the generation of singly and doubly methylated ditryptophan 2,5-diketopiperazines. Biochemistry 52:4274-4283. 
14. Cao L, Gurevich A, Alexander KL, Naman CB, Leão T, Glukhov E, Luzzatto-Knaan T, Vargas F, Quinn R, Bouslimani A, Nothias LF, Singh NK, Sanders JG, Benitez RAS, Thompson LR, Hamid M-N, Morton JT, Mikheenko A, Shlemov A, Korobeynikov A, Friedberg I, Knight R, Venkateswaran K, Gerwick WH, Gerwick L, Dorrestein PC, Pevzner PA, Mohimani H. 2019. MetaMiner: A scalable peptidogenomics approach for discovery of ribosomal peptide natural products with blind modifications from microbial communities. Cell Systems 9:600-608.e604.

15. Tietz JI, Schwalen CJ, Patel PS, Maxson T, Blair PM, Tai H-C, Zakai UI, Mitchell DA. 2017. A new genome-mining tool redefines the lasso peptide biosynthetic landscape. Nat Chem Biol 13:470-478.

16. Metelev M, Tietz JI, Melby JO, Blair PM, Zhu L, Livnat I, Severinov K, Mitchell DA. 2015. Structure, bioactivity, and resistance mechanism of streptomonomicin, an unusual lasso peptide from an understudied halophilic actinomycete. Chem Blol 22:241-250.

17. Kimura K, Kanou F, Takahashi H, Esumi Y, Uramoto M, Yoshihama M. 1997. Propeptin, a new inhibitor of prolyl endopeptidase produced by microbispora. I. Fermentation, isolation and biological properties. J Antibiot (Tokyo) 50:373-378.

18. Choi WS, Wu X, Choeng YH, Mahmud T, Jeong BC, Lee SH, Chang YK, Kim CJ, Hong SK. 2008. Genetic organization of the putative salbostatin biosynthetic gene cluster including the 2-epi-5-epi-valiolone synthase gene in Streptomyces albus atcc 21838. Appl Microbiol Biotechnol 80:637-645.

19. Schlorke O, Krastel P, Muller I, Uson I, Dettner K, Zeeck A. 2002. Structure and biosynthesis of cetoniacytone a, a cytotoxic aminocarba sugar produced by an endosymbiontic actinomyces. $J$ Antibiot (Tokyo) 55:635-642.

20. Wu X, Flatt PM, Xu H, Mahmud T. 2009. Biosynthetic gene cluster of cetoniacytone a, an unusual aminocyclitol from the endosymbiotic bacterium actinomyces sp. Lu 9419. Chembiochem 10:304314.

21. Heide L. 2009. The aminocoumarins: Biosynthesis and biology. Nat Prod Rep 26:1241-1250.

22. Kim CG, Lamichhane J, Song KI, Nguyen VD, Kim DH, Jeong TS, Kang SH, Kim KW, Maharjan J, Hong YS, Kang JS, Yoo JC, Lee JJ, Oh TJ, Liou K, Sohng JK. 2008. Biosynthesis of rubradirin as an ansamycin antibiotic from Streptomyces achromogenes var. Rubradiris nrrl3061. Arch Microbiol 189:463-473.

23. Castro JF, Razmilic V, Gomez-Escribano JP, Andrews B, Asenjo JA, Bibb MJ. 2015. Identification and heterologous expression of the chaxamycin biosynthesis gene cluster from Streptomyces leeuwenhoekii. App Environ Microbiol 81:5820.

24. Truman AW, Kwun MJ, Cheng J, Yang SH, Suh J-W, Hong H-J. 2014. Antibiotic resistance mechanisms inform discovery: Identification and characterization of a novel Amycolatopsis strain producing ristocetin. Antimicrob Agents Chemother 58:5687-5695.

25. Culp EJ, Waglechner N, Wang W, Fiebig-Comyn AA, Hsu YP, Koteva K, Sychantha D, Coombes BK, Van Nieuwenhze MS, Brun YV, Wright GD. 2020. Evolution-guided discovery of antibiotics that inhibit peptidoglycan remodelling. Nature 578:582-587.

26. Bode HB, Bethe B, Hofs R, Zeeck A. 2002. Big effects from small changes: Possible ways to explore nature's chemical diversity. Chembiochem 3:619-627.

27. Liu M, Grkovic T, Liu X, Han J, Zhang L, Quinn RJ. 2017. A systems approach using OSMAC, $\log p$ and NMR fingerprinting: An approach to novelty. Synth Syst Biotechnol 2:276-286.

28. Xu F, Wu Y, Zhang C, Davis KM, Moon K, Bushin LB, Seyedsayamdost MR. 2019. A genetics-free method for high-throughput discovery of cryptic microbial metabolites. Nat Chem Biol 15:161-168.

29. Amiri Moghaddam J, Crusemann M, Alanjary M, Harms H, Davila-Cespedes A, Blom J, Poehlein A, Ziemert N, Konig GM, Schaberle TF. 2018. Analysis of the genome and metabolome of marine myxobacteria reveals high potential for biosynthesis of novel specialized metabolites. Sci Rep 8:16600.

30. Bader CD, Panter F, Muller R. 2020. In depth natural product discovery - myxobacterial strains that provided multiple secondary metabolites. Biotechnol Adv 39:107480. 
31. Hernandez A, Nguyen LT, Dhakal R, Murphy BT. 2021. The need to innovate sample collection and library generation in microbial drug discovery: A focus on academia. Nat Prod Rep 38:292-300.

32. Jensen PR, Moore BS, Fenical W. 2015. The marine actinomycete genus Salinispora: A model organism for secondary metabolite discovery. Nat Prod Rep 32:738-751.

33. Steele AD, Teijaro CN, Yang D, Shen B. 2019. Leveraging a large microbial strain collection for natural product discovery. J Biol Chem 294:16567-16576.

34. Waglechner N, McArthur AG, Wright GD. 2019. Phylogenetic reconciliation reveals the natural history of glycopeptide antibiotic biosynthesis and resistance. Nat Microbiol 4:1862-1871.

35. Sun J, Zhao G, O'Connor RD, Davison JR, Bewley CA. 2021. Vertirhodins A-F, C-linked pyrrolidineiminosugar-containing pyranonaphthoquinones from Streptomyces sp. B15-008. Org Lett 23:682686.

36. Li H, Zhang M, Li H, Yu H, Chen S, Wu W, Sun P. 2021. Discovery of venturicidin congeners and identification of the biosynthetic gene cluster from Streptomyces sp. Nrrl s-4. J Nat Prod 84:110119.

37. Yang J, Song Y, Tang M-C, Li M, Deng J, Wong N-K, Ju J. 2021. Genome-directed discovery of tetrahydroisoquinolines from deep-sea derived Streptomyces niveus SCSIO 3406. J Org Chem doi:10.1021/acs.joc.1c00123.

38. Sharrar AM, Crits-Christoph A, Méheust R, Diamond S, Starr EP, Banfield JF. 2020. Bacterial secondary metabolite biosynthetic potential in soil varies with phylum, depth, and vegetation type. Mbio 11:e00416-00420.

39. Nayfach S, Roux S, Seshadri R, Udwary D, Varghese N, Schulz F, Wu D, Paez-Espino D, Chen IM, Huntemann M, Palaniappan K, Ladau J, Mukherjee S, Reddy TBK, Nielsen T, Kirton E, Faria JP, Edirisinghe JN, Henry CS, Jungbluth SP, Chivian D, Dehal P, Wood-Charlson EM, Arkin AP, Tringe SG, Visel A, Abreu H, Acinas SG, Allen E, Allen MA, Andersen G, Anesio AM, Attwood G, Avila-Magaña V, Badis Y, Bailey J, Baker B, Baldrian P, Barton HA, Beck DAC, Becraft ED, Beller HR, Beman JM, Bernier-Latmani R, Berry TD, Bertagnolli A, Bertilsson S, Bhatnagar JM, Bird JT, Blumer-Schuette SE, Bohannan B, Borton MA, Brady A, Brawley SH, Brodie J, Brown S, Brum JR, Brune A, Bryant DA, Buchan A, Buckley DH, Buongiorno J, Cadillo-Quiroz H, Caffrey SM, Campbell AN, Campbell B, Carr S, Carroll J, Cary SC, Cates AM, Cattolico RA, Cavicchioli R, Chistoserdova L, Coleman ML, Constant P, Conway JM, Mac Cormack WP, Crowe S, Crump B, Currie C, Daly R, Denef V, Denman SE, Desta A, Dionisi H, Dodsworth J, Dombrowski N, Donohue T, Dopson M, Driscoll T, Dunfield P, Dupont CL, Dynarski KA, Edgcomb V, Edwards EA, Elshahed MS, Figueroa I, Flood B, Fortney N, Fortunato CS, Francis C, Gachon CMM, Garcia SL, Gazitua MC, Gentry T, Gerwick L, Gharechahi J, Girguis P, Gladden J, Gradoville M, Grasby SE, Gravuer K, Grettenberger CL, Gruninger RJ, Guo J, Habteselassie MY, Hallam SJ, Hatzenpichler R, Hausmann B, Hazen TC, Hedlund B, Henny C, Herfort L, Hernandez $M$, Hershey OS, Hess M, Hollister EB, Hug LA, Hunt D, Jansson J, Jarett J, Kadnikov VV, Kelly C, Kelly R, Kelly W, Kerfeld CA, Kimbrel J, Klassen JL, Konstantinidis KT, Lee LL, Li W-J, Loder AJ, Loy A, Lozada M, MacGregor B, Magnabosco C, Maria da Silva A, McKay RM, McMahon K, McSweeney CS, Medina M, Meredith L, Mizzi J, Mock T, Momper L, Moran MA, Morgan-Lang C, Moser D, Muyzer G, Myrold D, Nash M, Nesb $\varnothing$ CL, Neumann AP, Neumann RB, Noguera D, Northen T, Norton J, Nowinski B, Nüsslein K, O'Malley MA, Oliveira RS, Maia de Oliveira V, Onstott T, Osvatic J, Ouyang Y, Pachiadaki M, Parnell J, Partida-Martinez LP, Peay KG, Pelletier D, Peng X, Pester M, Pett-Ridge J, Peura S, Pjevac P, Plominsky AM, Poehlein A, Pope PB, Ravin N, Redmond MC, Reiss R, Rich V, Rinke C, Rodrigues JLM, Rossmassler K, Sackett J, Salekdeh GH, Saleska S, Scarborough M, Schachtman D, Schadt CW, Schrenk M, Sczyrba A, Sengupta A, Setubal JC, Shade A, Sharp C, Sherman DH, Shubenkova OV, Sierra-Garcia IN, Simister R, Simon H, Sjöling S, Slonczewski J, Correa de Souza RS, Spear JR, Stegen JC, Stepanauskas R, Stewart F, Suen G, Sullivan M, Sumner D, Swan BK, Swingley W, Tarn J, Taylor GT, Teeling H, Tekere M, Teske A, Thomas T, Thrash C, Tiedje J, Ting CS, Tully B, 
Tyson G, Ulloa O, Valentine DL, Van Goethem MW, VanderGheynst J, Verbeke TJ, Vollmers J, Vuillemin A, Waldo NB, Walsh DA, Weimer BC, Whitman T, van der Wielen P, Wilkins M, Williams TJ, Woodcroft B, Woolet J, Wrighton K, Ye J, Young EB, Youssef NH, Yu FB, Zemskaya TI, Ziels R, Woyke T, Mouncey NJ, Ivanova NN, Kyrpides NC, Eloe-Fadrosh EA, Consortium IMD. 2020. A genomic catalog of earth's microbiomes. Nature Biotech doi:10.1038/s41587-020-0718-6.

40. Koren S, Walenz BP, Berlin K, Miller JR, Bergman NH, Phillippy AM. 2017. Canu: Scalable and accurate long-read assembly via adaptive k-mer weighting and repeat separation. Genome Res 27:722-736.

41. Vaser R, Sović I, Nagarajan N, Šikić M. 2017. Fast and accurate de novo genome assembly from long uncorrected reads. Genome Res 27:737-746.

42. Gurevich A, Saveliev V, Vyahhi N, Tesler G. 2013. Quast: Quality assessment tool for genome assemblies. Bioinformatics 29:1072-1075.

43. Li H. 2018. Minimap2: Pairwise alignment for nucleotide sequences. Bioinformatics 34:3094-3100.

44. Gatto L, Gibb S, Rainer J. 2021. MSnbase, efficient and elegant r-based processing and visualization of raw mass spectrometry data. J Prot Res 20:1063-1069.

45. O'Boyle NM, Banck M, James CA, Morley C, Vandermeersch T, Hutchison GR. 2011. Open Babel: An open chemical toolbox. J Cheminformatics 3:33. 